\title{
Some Replenishments with the Study on Motility Changes of Dictyostelium discoideum Pseudoplasmodium under Light Condition
}

\author{
Masanobu Kitami \\ Department of Physics, Faculty of Education, Yokohama National University, \\ Tokiwadai 156, Hodogaya-ku, Yokohama, 240 Japan
}

Received January 14, 1983

It has been well known that the cells of $D$. discoideum show collective motion characterized as migration at the pseudoplasmodial stage. The pseudoplasmodium (slug) migrates while secreting from its surface the slime sheath, which is left behind as the slug moves on. In addition, the migrating slug exhibits an extremely sensitive phototactic response (Bonner et al. 1950, Poff and Loomis 1973, Poff et al. 1973, 1974). In phototactic migration, the basis of the oriented migration is a turning movement of the slug, initiated by the turning of the anterior tip toward the light source (Poff and Loomis 1973). This mechanism of turning is similar in concept to the following phenomenon: "A tracked vehicle makes a turn by the difference in speed between the outside and inside tracks" (Loomis 1975).

Some experimental observations and hypothetical models have been established in order to explain the mechanism of slug migration (Bonner et al. 1950, Durston snd Vork 1979, Francis 1964, Häder and Poff 1979a, 1979b, Loomis 1972, Maeda 1977, Poff and Loomis 1973, Poff et al. 1973, 1974). Nevertheless, since no conclusive evidence is available on the slug migration itself nor on its oriented migration, it is not elucidated whether it is the slime sheath or the interior cells that control such collective motion as migration.

Therefore, it is essential to measure the motive force of the migrating slug as one of the means to study the slug migration and the mechanism of its oriented migration (Bonner et al. 1953, Inouye and Takeuchi 1980, Loomis 1975, Yamamoto and Kamiya 1971).

In the previous report, the maximum motive force of the migrating slug of $D$. discoideum in the dark condition was measured as $0.6-1.6$ dyn by using an original method utilizing the centrifugal force, which investigates quantitatively the motility of the migrating slug (Kitami 1982). It was also reported that this centrifugal method was an effectual measure even for the maximum motive force of the slug in phototactic migration. The maximum value of it was $0.8-2.4$ dyn, which was nearly 1.5 times larger than that in the dark. Furthermore, some interesting results were obtained by measuring changes in the velocity of the slugs (see below) (Kitami 1982).

The present report discusses, in consequence of more detailed considerations on the results of the motility changes of slug, a possibility that changes in the motility of interior cells in both sides of the slug, induced by illumination, directly 
participate in the turning response of it during the phototactic migration.

Summary of the results of the motility changes of slug

D. discoideum slug (NC4) was used throughout. The experiment was designed to measure the changes in the velocity of the slugs placed under two opposite conditions, i.e., combinations of illuminated from the front or the back, and migrating in the direction of or in the opposite direction to the applied centrifugal force.

Fig. 1 shows the dependence of the velocity of the migrating slugs upon the varying strengths of the centrifugal force under two opposite conditions: i) Slugs

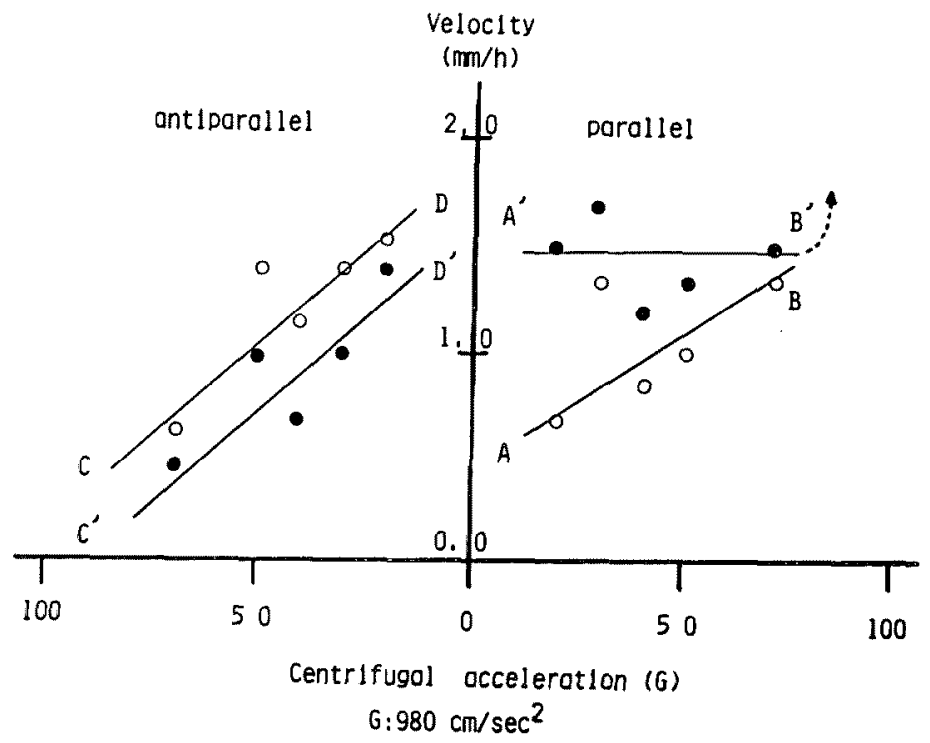

Fig. 1. Dependence of the velocities of the migrating slugs (ordinate) upon varying strengths of the centrifugal force (abscissa) in the nuit of $G=980 \mathrm{~cm} / \mathrm{S}^{2}$ in the dark ( ) or light $(0)$. The individual points represent the mean velocity calculated by collecting data from two or three most rapidly migrating slugs in each plate of four or five runs. i) Solid line A-B; slugs were migrating parallel to the direction of the centrifugal force with exposure to light from the back. ii) Solid line C-D; they were migrating antiparallel to it with exposure to ligth from the front. To compare with the data under illuminated condition, the data, i.e., parallel to it in the dark (solid line $\mathrm{A}^{\prime}-\mathrm{B}^{\prime}$ ) and antiparallel to it in the dark (solid line $\mathrm{C}^{\prime}-\mathrm{D}^{\prime}$ ) are also shown.

were migrating parallel to the direction of the centrifugal force with exposure to light from the back (solid line A-B). ii) They were migrating antiparallel to it wilh exposure to light from the front (solid line C-D). The data in the dark condition are also shown in order to compare with the data in the illuminated condition, i.e., they were migrating parallel to it in the dark (solid line $\mathrm{A}^{\prime}-\mathrm{B}^{\prime}$ ) and antiparallel to it in the dark (solid line $\mathrm{C}^{\prime}-\mathrm{D}^{\prime}$ ).

i) Illuminating the slugs from the back usually results in a turning response of the migraion toward the light source. But, from these data, it was adopted that the only slugs continued the migration in the same direction as before without turning. The velocities of the slugs migrating parallel to the centrifugal force with ex- 
posure to light from the back, were undoubtedly dependent upon the applied centrifugal force (see solid line A-B). Moreover, the velocities were slower than in the dark. It could be concluded therefore, that the motion of the migrating slug was affected by illumination from the back.

ii) The velocities of the slugs migrating toward the light source in antiparallel direction to the centrifugal force were undoubtedly dependent upon the applied centrifugal force, as had been expected, and they were larger than those in the dark (see solid line C-D). The maximum motive force of the slug in phototactic migration was estimated from these data. The extrapolated value of the applied centrifugal force (the acceleration of the centrifugal motion) to the velocity zero was about $120 \mathrm{G}$ (see point $\mathrm{C}$ in Fig. 1). So, the maximum value of the motive force of the slug in phototactic migration was obtained as $0.8-2.4$ dyn, which was nearly 1.5 times larger than the value in the dark.

When the slugs exposed to light were compared with those in the dark, it was clear that illumination directly influenced the motive force because illumination from the front increased their velocities and the increment above those in the dark was nearly constant (see solid lines $\mathrm{C}-\mathrm{D}$ and $\mathrm{C}^{\prime}-\mathrm{D}^{\prime}$ for comparison). On the contrary, illumination from the back caused quite opposite results; i.e., velocities were decreased and the decrements, compared with those in the dark, depended strongly upon the centrifugal force applied to them (see solid lines $\mathrm{A}-\mathrm{B}$ and $\mathrm{A}^{\prime}-\mathrm{B}^{\prime}$ for comparison).

\section{Discussion}

As described before, the turning response of the slug toward the light source has generally been taken to be the following: The illumination on the unilateral side of the slug is focused onto the distal side by the "lens effect", inducing difference in light intensity between two sides of it (Francis 1964, Poff and Loomis 1973). This probably brings about the velocity difference in two sides (Francis 1964). At present, there are, at least, two possibilities to give rise to this velocity difference; a) it is probably induced by the difference in speed of the slime sheath production in both sides (Poff and Loomis 1973) or b) recent studies have been reported that movement of each $D$. discoideum amoebae is responsive to light (Häder and Poff $1979 \mathrm{a}, 1979 \mathrm{~b}$ ), suggesting a possibility of direct effect of illumination of the difference in the motility of cells in both sides of the migrating slug.

On the basis of the present results described above, there is no doubt that illumination from both the front and the back directly influences the motility of the migrating slugs. As far the result (ii), possible reasons, at least, for this increment seem to be both A) because of changes in the slime sheath production and B) because of changes in the motility of the interior cells, those may be mainly stimulated at the anterior part of the migrating slug. Therefore, although the motive force of the slug in phototactic migration can be estimated, conclusive information to make us determine either of the two reasons mentioned above has not been taken out from these data.

On the other hand, result (i) seems to be of great interest. As the conditions of the applied centrifugal force in both cases (solid lines: A-B and $\mathrm{A}^{\prime}-\mathrm{B}^{\prime}$ ) were the same, 
this decrement was obviously caused by only illumination from the back. Moreover, this effect of illumination from the back seemed to directly affect its motive force and /or mechanism of its migration, since the decrements were large when the applied centrifugal force was small (see points $\mathrm{A}$ and $\mathrm{A}^{\prime}$ for comparison) and they got smaller with an increase in the centrifugal force.

Considering the cause of the decreasing velocity of the slug in this case only from the view point of the changes in the speed of the slime sheath production, it should be reduced by the influence of illumination from the back, contrary to the influence of illumination from the front (see above (A)).

As mentioned above, in order to explain the increase or decrease of the velocity of the slug in these two cases, from the view point of the changes in speed of the slime sheath production, the following hypothesis will be required: Speed of the slime sheath production of the slug in phototactic migration will change according to the "direction", i.e., from the front or the back, of illumination toward it. In other words, quantity of secretion of the slime sheath from each cell in the slug depends on the "direction" of light toward the interior cells of it. However, this hypothesis cannot be acknowledged so readily, and no report has been given in favor of this hypothesis.

Moreover, regarding the phototactic response of the slug which seems to be mediated by phototaxin in the interior cell (Poff et al. 1974), it is likely that, through photo-oxidation of phototaxin, quantity (speed) of the slime sheath production depends on the intensity and/or the wave-length of the incident light; however, it is unlikely that quantity (speed) of its secretion depends only on the applied force whose effect is purely physical as the results of this experiment.

By the way, the motive force of the migrating slug is possibly brought about by the interior cells of it, since the contractile protein exists in the motile $D$. discoideum cell (Clarke et al. 1975, Eckert et al. 1977). Therefore, changes in velocity of the slug in the dark condition, which correspond to the strength of the centrifugal force, are possibly interpreted as changes in the motility of the interior cells within the limitation of speed of the slime sheath production of the slug under a certain situation (Kitami 1982).

Even in the light condition, it is quite possible that illumination directly influence the motility of the interior cells of it, considering that changes in the velocity of the slug strongly depends upon the applied centrifugal force and that it is likely to be impossible to explain the reason for the changes in the velocity of the slug only from the view point of changes in the slime sheath production.

The author has shown, from some indirect evidences, that the main resson for inducing changes in the velocity of the slug under illuminated condition from those in the dark is not because of changes in the speed of the slime sheath production but because of changes in the motility of the interior cells of the migrating slug owing to receive the direct influence of illumination.

Although it is an open question about the direct influence of light upon motility of the interior cells, the results in the present study strongly suggest a considerable possibility that the difference in the velocity which probably occurrs in both sides of the slug, during its turning response (phototaxis), is controlled not by the difference 
in "passive" movenent of the interior cells induced by the slime sheath (Poff and Loomis 1973) but mainly by the difference in "active" movement of the interior cells themselves in both sides of it, which is induced by illumination toward them.

Recently, Smith and others (1982) proposed that the orientation of D. discoideum slug was not mediated by a sustained changed in slug speed and that slug movement was not directly coupled to tactic responses. However, it seems to be impossible to dismiss the possibility that difference in motility of the interior cells directly participates in the changes in the slug movenent. Because, the suggestion in the present study has been supported by the fact that difference in speed of the interior cells in both sides of the slug, which shows the turning response induced by unilateral light, was observed by a cinematographical study (Yamada et al. 1981).

\section{References}

Bonner, J. T. 1947. Evidence for the formation of cell aggregates by chemotaxis in the development of the slime mold D. discoideum. J. Exp. Zool. 106: 1-26.

-, Clark, W. W., Neely, C. L. Jr. and Slifkin, M. K. 1950. The orientation to light and extremely sensitive orientation to temperature gradients in the slime mold $D$. discoideum. J. Cell Comp. Physiol. 36: 149-158.

-, Koontz, P. G. and D. Paton. 1953. Size in relation of the rate of migration in the slime mold Dictyostelium discoideum. Mycoologia 45: 235-240.

Clarke, M., Schatten, G., Mazia, D. and Spudich, J. A. 1975. Visualization of actin fibers associated with the cell membrane in amoebae of Dictyostelium discoideum. Proc. Nat. Acad. Sci. U.S.A. 72: 1758-1762.

Durston, A. J. and Vork, F. 1979. A cinematographical study of the development of vitally stained Dictyostelium discoideum. J. Cell Sci. 36: 261-279.

Eckert, B.S., Warren, R. H. and Rubin, R.W. 1977. Structual and biochemical aspects of cell motility in amebas of Dicctyostelium discoideum. J. Cell Biol. 72: 339-350.

Francis, D. W. 1964. Some studies on phototaxis of Dictyostelium. J. Cell Comp. Physiol. 64: 131-138.

Häder, D-P and Poff, K. 1979a. Light-induced accumulation of Dictyostelium discoideum amoebae. Photochem. and Photobiol. 29: 1157-1162.

- and - 1979b. Photodispersal from lighttfraps by amoebae of Dictyostelium discoideum. Exp. Mycol. 3: 121-131.

Inouye, K. and Takeuchi, I. 1980. Motive force of the migrating pseudoplasmodium of the cellular slime mould Dictyostelium discoideum. J. Cell Sci. 41: 53-64.

Kitami, M. 1982. The motive force of the migrating pseudoplasmodium of Dictyosteluim discoideum under dark and light conditions. J. Cell Sci. 56: 131-140.

Loomis, W. F. 1972. Role of the surface sheath in the control of morphogenesis on Dictyostelium discoideum. Nature, New Biol. 240: 6-9.

- 1975. (ed.) Dictyostelium discoideum: A Developmental System. Academic Press, New York.

Maeda, Y. 1977. Role of cyclic AMP in the polarized movement of the migrating pseudoplasmodium of Dictyostelium discoideum. Develop. Growth and Differ. 19: 201-205.

Poff, K. and Loomis, W. F. 1973. Light induced absorbance change associated with phototaxis in D. discoideum. Exp. Cell Res. 82: 236-240.

-, Butler, W. and Loomis, W. F. 1973. Control of phototactic migration in Dictyostelium discoideum. Proc. Nat. Acad. Sci. U. S. A. 70: 813-816.

-, Loomis, W. F. and Butler, W. 1974. Isolation and purification of the photoreceptor pigment associated with phototaxis in Dictyostelium discoideum. J. Biol. Chem. 249: 2164-2168.

Smith, E., Fisher, P. R., Grant, W. W. and Williams, K. L. 1982. Sensory behaviour in Dictyostelium discoideum slug: phototaxis and thermotaxis are not mediated by a change in slug speed. 
J. Cell Sci. 54: 329-339.

Yamada, T., Ishii, K. and Higuchi, G. 1981. Proceedings of the 46th Annual Meeting of the Botanical Society of Japan. 46: 129 (in Japanese).

Yamamoto, M. and Kamiya, N. 1971. On the mechanism of slug movement of Dictyostelium dis. coideum. Japanese J. Develop. Biol, 25: 84-85 (in Japanese). 